\title{
Competencias del estudiante de enfermería en la promoción y detección de la tuberculosis pediátrica
}

\author{
Lisseth Karolina Chicas Blanco ${ }^{1}$ \\ Abraham Hernández Rivera ${ }^{2}$ \\ Úrsula Guadalupe Rodríguez ${ }^{3}$ \\ Docentes investigadores, Facultad de Ciencias de la Salud \\ Universidad Católica de El Salvador, El Salvador
}

Fecha de recepción: 09-06-2020

Fecha de aceptación: 09-01-2021

\section{Resumen}

La tuberculosis es una de las principales causas de muerte en población pediátrica a nivel mundial, aun cuando es una enfermedad curable, muchos niños con tuberculosis no son diagnosticados de manera oportuna y como consecuencia de ello no reciben el tratamiento que podría salvarles la vida. Por lo tanto, se hace indispensable contar con personal altamente capacitado para generar una efectividad en las intervenciones de promoción y detección de la tuberculosis pediátrica, por ello es que el rol del estudiante de enfermería en servicio social juega un papel transcendental para llevar acabo dichas intervenciones.

El estudio se focalizó en identificar las potencialidades de los estudiantes en servicio social de Licenciatura de Enfermería para la promoción y detección de tuberculosis en niños menores de 10 años, el enfoque del estudio fue cuantitativo, bajo una investigación básica. El objeto de estudio fueron 38 estudiantes de Licenciatura en Enfermería que realizan su servicio social en 4 hospitales y 14 Unidades Comunitarias de Salud Familiar de la zona occidental; y para recolectar datos se administró un cuestionario estructurado.

Los datos sintetizados arrojaron la necesidad de fortalecer los conocimientos relacionados a prevención de tuberculosis infantil, también se detectó que existe necesidad de generar estrategias de promoción y detección de esta enfermedad, puesto que solo un 39\% de los estudiantes las realizan en campo práctico. Por lo tanto, es de suma importancia mediar el conocimiento en materia de tuberculosis pediátrica en los futuros prestadores de salud en Enfermería.

Palabras clave: Tuberculosis pediátrica, estudiante en servicio social, potencialidades, enfermería, prevención y promoción.

\begin{abstract}
Tuberculosis is one of the leading causes of death in the pediatric population worldwide, even though it is a curable disease, many children with tuberculosis are not diagnosed in a timely manner and as a consequence do not receive the treatment that could save their lives. Therefore, it is essential to have highly trained personnel to generate effective interventions for the promotion and detection of pediatric tuberculosis, which is why the role of the nursing student in social service plays a transcendental role to carry out such interventions.

This study focused on identifying the potentialities of students in social service of the Nursing Degree for the promotion and detection of tuberculosis in children under 10 years of age, the focus of the study was quantitative, under a basic investigation. The study subjects were 38 Nursing Bachelor students carrying out their social service in 4 hospitals and 14 Community Family Health Units in the western area; and to collect data, a structured questionnaire was administered.

The synthesized data showed the need to strengthen knowledge related to childhood tuberculosis prevention, it was also identified a need to generate strategies for the promotion and detection of this disease, since only $39 \%$ of the students carry them out in a practical field. Therefore, it is extremely important to mediate knowledge about pediatric tuberculosis in future health providers in Nursing.
\end{abstract}

Key words: Pediatric tuberculosis, social service student, potential, nursing, prevention and promotion.

1. Maestra en Asesoría Educativa; docente investigador; email: lisseth.chicas2@catolica.edu.sv; ORCID: https://orcid.org/00000002-3833-1898

2. Maestro en Asesoría Educativa; docente investigador; email:abrarive2013@gmail.com; ORCID: https://orcid.org/0000-0002$6261-1208$

3. Maestra en Asesoría Educativa; docente investigador; email: ursula.rodriguez@catolica.edu.sv; ORCID: https://orcid.org/00000002-9794-3819 


\section{Introducción}

Es primordial que el estudiante egresado de la carrera de licenciatura en enfermería obtenga las potencialidades necesarias para la promoción y detección oportuna de la enfermedad de la tuberculosis (TB) 4 , ya que es considerada "una de las diez primeras causas de muerte en población pediátrica en todo el mundo" (Ministerio de Salud [MINSAL]5, 2021, p. 8). Muchos niños con TB no son diagnosticados y como consecuencia de ello no reciben el tratamiento que podría salvarles la vida, por lo anterior, el MINSAL (2020a) describe que:

Diferentes instituciones de Educación Superior de profesionales en enfermería del país, pueden desarrollar un papel decisivo en el abordaje integral del problema desde la educación para la salud en los usuarios hasta la atención directa e integral; debido a que la tuberculosis es una enfermedad infecto contagiosa, se deben ejecutar acciones de promoción, prevención, diagnóstico y tratamiento, para lograr la integración y participación en forma persistente y sistemática de la población. (p.11)

Entre las competencias que el egresado de Licenciatura de Enfermería debe desarrollar sobre el programa de Tuberculosis en el primer nivel están.

- Participación en la detección del sintomático respiratorio
- Aplicación de la vacuna BCG en el recién nacido

- Aplicación de precipitado proteico (PPD)

- Estudio de contactos

- Administración de quimioprofilaxis

- Promoción de la salud en el programa

- Orientación en la toma de muestras de esputo

- Administración del tratamiento antituberculoso estrictamente supervisado

- Detección de efectos colaterales del usuario con tratamiento antituberculoso

- Referir al médico al usuario para evaluación y tratamiento de las reacciones adversas

- Consejería al usuario con tuberculosis

- Visita domiciliar (Para realizar el diagnóstico de las condiciones en que vive el paciente con tuberculosis)

- Manejo del sistema de información y registro del estudio de cohorte

- Evaluaciones del programa (Chanta, J., comunicación personal, 21 de agosto 2018).

Además, en el segundo nivel de atención, en su mayoría, hospitales básicos, es el personal de enfermería quien realiza procedimientos de aspirado gástrico, toma de esputo inducido para la recolección de muestras de baciloscopías o cultivos para el estudio de Tuberculosis en los niños; “estas muestras deben ser obtenidas por personal médico o de enfermería

4. En adelante, los autores harán referencia al término mediante su acrónimo.

5. Dentro del documento, los autores harán referencia a la institución mediante su acrónimo. 
entrenado, y para evitar demoras en el procesamiento" (MINSAL, 2017, p.31).

En la Universidad Católica de El Salvador, en el transcurso de la formación académica de enfermería se imparten a los estudiantes los componentes necesarios del programa de tuberculosis para el desarrollo de potencialidades cognitivas y técnicas, de forma escalonada, incluyendo la atención al adulto, a la mujer en estado de embarazo y niños.

No obstante, existen brechas educativas que definir e identificar haciendo énfasis primordialmente en el componente de la promoción de la salud y prevención de la enfermedad de TB. Una de las prioridades de MINSAL (2015) es:

Incrementar el conocimiento sobre las condiciones que intervienen, favorables y desfavorables, en la detección de los casos de $\mathrm{TB}$, en los diferentes niveles de atención del Sistema Nacional de Salud, para diseñar estrategias de intervención en la detección temprana de TB como actividad coordinada con instituciones formadoras de recursos de salud y sus estudiantes para fortalecer a través del educando próximo a graduarse, las estrategias diferenciadas de abordaje, basadas en la revisión detallada de las brechas de detección de sintomático respiratorio $(\mathrm{SR}) \mathrm{y}$ diagnóstico de casos. (p.72)

La promoción para la salud encaminada a los cambios de los estilos de vida de las personas para el mejoramiento de la salud, se realiza desde el primer nivel de atención en salud. Por tanto, los estudiantes en servicio social realizan actividades de educación para la salud, fortaleciendo así los mecanismos de autocuidado en la población al recibir información sobre formas de transmisión de la enfermedad de la TB e indirectamente, beneficiando a la niñez; MINSAL (2020b), hace el siguiente énfasis:

El personal de salud, debe realizar promoción de la salud, para crear condiciones saludables a través de acciones concretas, desarrollar habilidades personales y generar mecanismos administrativos, organizativos y políticos, que faciliten a las personas y grupos poblacionales tener mayor control sobre su salud y mejorarla permanentemente. (p. 11)

En ese contexto es necesario que el estudiante tenga conocimientos, habilidades y destrezas para ejecutar las diferentes actividades de promoción para la salud dirigidas cortar la cadena de transmisión de la tuberculosis en la niñez. En el país, cada año se detectan personas con VIH, siendo los niños un contacto vulnerable por la coinfección TB/ VIH. "Para el año 2019 en El Salvador se detectó a 1161 personas con VIH" (MINSAL, 2019a).

La población debe recibir información sobre signos y síntomas de la tuberculosis para la detección oportuna de síntomas respiratorios por el riesgo de transmisión a la población vulnerable, como es el caso de la niñez. Tanto el profesional, como el estudiante de enfermería, deben aplicar todas las actividades encaminadas a la prevención de la TB en conjunto con el equipo de salud con la finalidad de evitar la infección. 
Así mismo, "el estudiante de enfermería debe realizar intervenciones dirigidas a evitar la infección por el Mycobacterium tuberculosis, en las cuales se incluye la detección y diagnóstico precoz de la tuberculosis pediátrica" (MINSAL, 2020a, p. 16).

Además, el estudiante de enfermería debe considerar los aspectos siguientes:

1. Los niños en contacto con una persona adulta con tuberculosis, dada la susceptibilidad a la infección y enfermedad tuberculosa, deben ser referidos a la Unidad Comunitaria de Salud Familiar (UCSF) ${ }^{6}$, para ser examinados e investigados, o de ser necesario al segundo o tercer nivel de atención, para evaluación por médico pediatra.

2. Los niños considerados como contacto intra domiciliar del caso de TB deben recibir consulta médica y se les debe indicar los estudios de laboratorio y de gabinete necesarios para descartar la enfermedad.

3. Orientar al grupo familiar para prevenir la enfermedad principalmente en medidas como:

- Taparse la boca al toser o estornudar (etiqueta de la tos).

- Evitar el hacinamiento, en la medida de lo posible.

- Mejorar la ventilación de las habitaciones.

- Consejería sobre alimentación balanceada. (MINSAL, 2020a, p. 16)
Cabe destacar la importancia de la detección oportuna de sintomáticos respiratorios, ya que de esta forma se contribuye de manera indirecta para la prevención de los contactos TB menores de 10 años.

\section{Procedimientos de enfermería para la detec- ción y control de tuberculosis en menores de 10 años}

Los estudiantes de enfermería aplican los conocimientos en la ejecución de sus prácticas, integrándose al equipo de salud al participar en la atención del paciente pediátrico con tuberculosis, por lo que se espera que sus competencias cognitivas y prácticas mejoren en la toma y reporte oportuno de muestras para el diagnóstico de la tuberculosis en niños.

En ese contexto, el estudiante de enfermería asume un papel importante en el diagnóstico de la tuberculosis en la niñez, ya que realiza procedimientos para la recolección de muestras de niños menores de 10 años. Los métodos diagnósticos aprobados por el MINSAL y que son aplicadas por el estudiante de enfermería son las siguientes:

a. Prueba de esputo inducido. Esta se obtiene por aspiración nasofaríngea; previo a nebulizaciones, no es invasiva y no requiere ingreso. Por la naturaleza de la prueba es necesario que el estudiante tenga las competencias técnicas para que la muestra sea de calidad.

b. Aspirado gástrico. Se debe emplear especialmente en niños que no pueden expec-

6. Dentro del documento, los autores harán referencia a la institución mediante su acrónimo. 
torar. El estudiante debe realizar el procedimiento de colocación de sonda nasogástrica y saber cuánto esputo debe aspirar, por lo que debe conocer la preparación del paciente para que la muestra sea de calidad.

c. Lavado gástrico. Una vez que la sonda está en el estómago y no se pudo obtener la cantidad de muestra en el aspirado gástrico, es necesario recurrir al lavado gástrico, por lo que el estudiante debe saber realizar este procedimiento. (MINSAL, 2017, p.28)

Debido a lo anterior, los estudiantes de enfermería del pregrado son capacitados previo a su servicio social de forma permanente con base a las actualizaciones de los Instrumentos Técnico Jurídicos del Programa Nacional de Tuberculosis y Enfermedades Respiratorias (PNTYER), el cual es considerado como:

Una instancia del Ministerio de Salud, responsable de definir las directrices, estrategias, políticas y acciones relacionadas a la promoción, prevención y control de la Tuberculosis y las enfermedades respiratorias, orienta esfuerzos para la capacitación y actualización del recurso humano [...]. Asesora a las instituciones formadoras de recursos para que transmitan el conocimiento del problema de la tuberculosis a nivel académico, pregrado, post grado y de educación continua. (MINSAL, 2019b, p.1)

En ese marco, este estudio permitió la identificación de las potencialidades que poseen los estudiantes de enfermería en servicio social en relación a la promoción y detección de la infección de tuberculosis en niños en UCSF y en hospitales de segundo nivel.

\section{Metodología}

El estudio que se realizó consistió en una investigación básica con un enfoque cuantitativo que es aplicado "cuando utiliza estadísticas, analiza la realidad objetiva" (Hernández, 2014, p.36). De tipo descriptivo puesto que se describen las competencias de los estudiantes en materia de promoción y detección de la tuberculosis pediátrica.

Para el estudio de las variables se tomó en cuenta un estudio de tipo transversal, puesto que fueron estudiadas de manera simultánea y tomando en cuenta la temporalidad de la investigación fue de tipo prospectivo.

La población y muestra estuvo constituida por 38 estudiantes y 20 jefaturas de Enfermería, para valorar la veracidad de los datos emitidos por los estudiantes. Se realizó en 14 UCSF y 4 hospitales de la zona occidental.

Los objetivos fueron la base para planificar asertivamente la recolección de datos. Por tanto, las técnicas empleadas fueron la encuesta con su respectivo instrumento, el cuestionario, el cual se administró a los 38 estudiantes en servicio social; y para jefaturas y supervisoras locales se aplicó la técnica de entrevista con el instrumento de guía de entrevista estructurada. A los participantes, se les detallaron los procedimientos para garantizar los aspectos éticos, a través de un formato de consentimiento informado. 


\section{Resultados}

\section{Promoción}

Las dificultades diagnósticas del niño y la falta de formulaciones pediátricas óptimas son el mayor desafío para control de TB infantil (Mellado et al., 2017, p.51). Por lo que el estudiante, debe conocer y afrontar oportunamente este desafío generando estrategias en la promoción y detección en su práctica y desempeño profesional; debido a que: "Todo niño que vive o pasa tiempo en un entorno donde existen personas con TB infecciosa podría estar expuesto al Mycobacterium tuberculosis" (OMS, 2016, p. 2).

En cuanto al conocimiento general de la tuberculosis pediátrica, se identificó con lente crítico que el $40 \%$ de los encuestados desconoce sobre tan importante temática, lo que implica falencias en los procesos de promoción y detección de la TB pediátrica, alterando la calidad de atención en este grupo etario.

Los datos también arrojaron que un 39\% de la población realiza acciones de promoción y detección de tuberculosis y el $61 \%$ de la población no realiza ninguna acción de promoción y detección para la TB pediátrica, dato que es relevante para generar estrategias educativas que conlleven a una concientización sobre la importancia de cultivar en los estudiantes la necesidad de brindar una atención de calidad a grupos vulnerables. También, se observó que este dato tiene relación íntima con el $40 \%$ de los estudiantes que desconocen sobre el tema de la TB pediátrica.
En el desarrollo del servicio social, como parte de las funciones en UCSF, está la búsqueda activa de casos de niños contacto TB confirmados, en la cual participa el personal de salud encargado del programa y los estudiantes cuando están en UCSF. Sin embargo, los resultados señalaron que el 55\% de los estudiantes no ha participado en ello, pero debe tomarse en cuenta que del total de la población en estudio un $52 \%$ realizó su servicio social en áreas hospitalarias, donde no se tienen funciones de búsqueda de casos. Por lo que el dato de esta variable estudiada refleja que el $45 \%$ de estudiantes sí ha buscado contactos TB en niños en las diferentes edades entre 1 y 10 años.

El paciente bacteriológicamente confirmado, inicia Tratamiento Acortado Estrictamente Supervisado (TAES), el personal de enfermería encargado del programa, entrega cada día los antifímicos al paciente, verifica su ingesta y da consejerías. Sin embargo, el 53\% de los estudiantes dijo desconocer, lo que pone en riesgo los contactos de este caso índice tratado.

Por tanto, la relevancia de la prevención y la necesidad que el estudiante se interese en la búsqueda activa de casos nuevos de TB en niños, se observó en los resultados de la entrevista a jefaturas inmediatas de los estudiantes, ya que solamente un $28 \%$ de ellos se interesan en la búsqueda de los contactos de casos índice, labor que se puede realizar, tanto en las UCSF y en hospitales de segundo nivel.

Previo a la realización de un procedimiento de enfermería, el estudiante ya ha recibido el com- 
ponente teórico y científico; pero los resultados señalaron que solo el $46 \%$ de los estudiantes recuerdan el componente teórico, quienes identificaron la respuesta correcta del medio de diagnóstico para estudio bacteriológico en el menor de 10 años para la detección de la Tuberculosis pediátrica (ver figura 1).

Otra de las pruebas que se realizan en los niños es la prueba cutánea de derivado proetico purificado (PPD por sus siglas en inglés) ${ }^{7}$ a través del conocimiento operativo de la aplicación de la prueba y lectura de la misma, y el $34 \%$ de los estudiantes respondió que no todos los educandos están preparados en cuanto a conocimientos operativos sobre esta prueba, la cual es parte del diagnóstico de TB en niños en el MINSAL.
Con base en la respuesta de las jefaturas en cuanto a la participación de los estudiantes en la toma de muestras diagnósticas en niños, se determinó que solamente un $22 \%$ de los estudiantes logró ser partícipe por lo menos algún caso. Esto tomando en cuenta que no todos los estudiantes se encontraban en instituciones de salud donde se realizan todas las pruebas invasivas, a diferencia de la PPD que si es tomada tanto en hospitales, como en UCSF, lo que genera mayor probabilidad de participación a quienes están asignados a hospitales, específicamente en pediatría.

\section{Discusión}

El conocimiento sobre la promoción y detección de la tuberculosis pediátrica es de suma importancia para incidir en el corte de la cade-

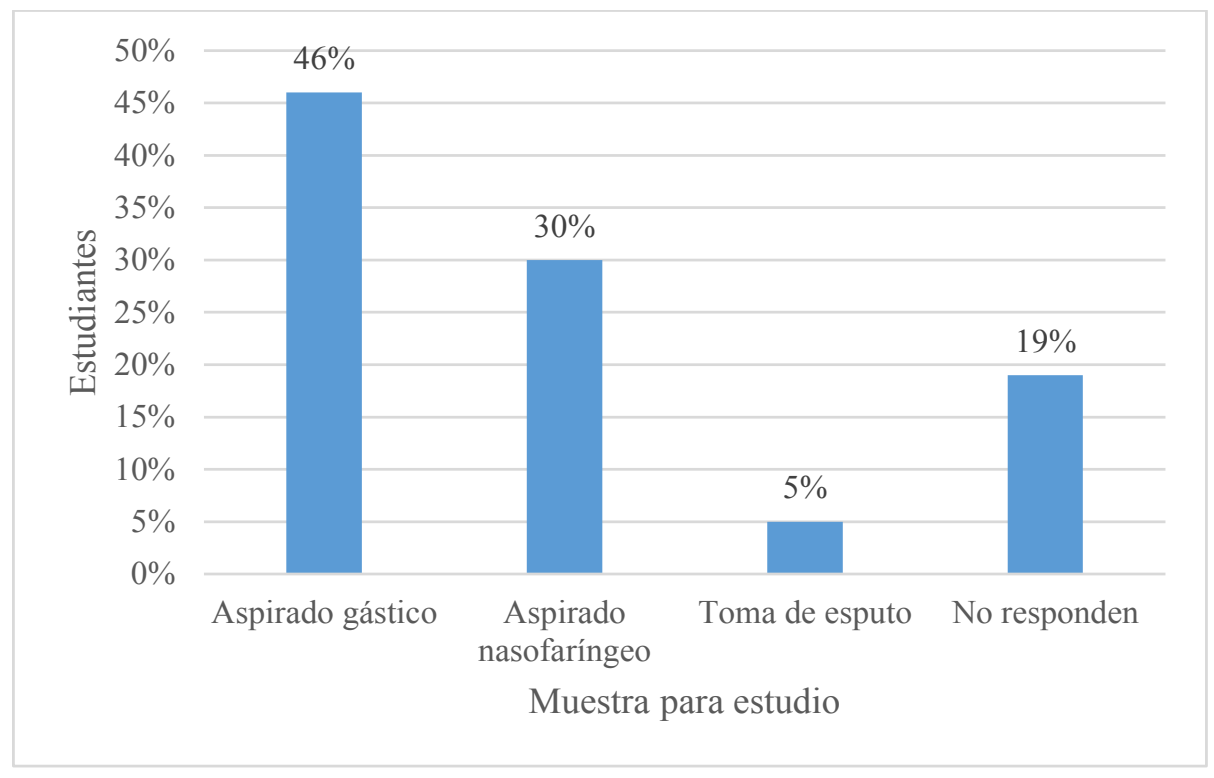

Figura 1. Identificación del procedimiento diagnóstico para estudio bacteriológico en niños contacto TB o sospechoso menor de 10 años.

7. Dentro del documento, los autores harán referencia a esta prueba mediante sus siglas en inglés. 
na de transmisión de la infección, en este sentido, el estudiante de enfermería debe contar con las competencias cognitivas y prácticas que le permitan realizar las actividades de educación en salud utilizando las estrategias de información, educación y comunicación que logren la modificación de conductas de los usuarios sobre la prevención de la tuberculosis. En ese marco, el 40\% de los estudiantes mostró deficiencia en los conocimientos generales sobre la tuberculosis pediátrica. Esta situación puede incidir en la omisión de actividades de promoción y detección por falta de conocimientos. Esta falta de conocimientos se refleja también en el abordaje de la consejería que el estudiante debe dar al paciente con tuberculosis, ya que el $53 \%$ desconoce los puntos que se deben impartir en esta actividad educativa, sobre todo en lo relevante al aislamiento que debe haber para la prevención de la TB pediátrica.

De esa misma manera, el hecho de que el 61\% de los estudiantes no realicen acciones de promoción es un llamado de atención para que el establecimiento dé estrategias que permitan que el estudiante pueda desempeñar un rol activo en esta área del programa de Prevención y Control de la Tuberculosis.

En cuanto a la detección, es importante que el estudiante no solo tenga las competencias cognitivas, sino también las competencias técnicas para que este pueda realizar los procedimientos que van incluidos en la obtención de muestras en los niños menores de 10 años. El 45\% de los estudiantes tuvo la oportunidad de participar en la obtención de muestras, demostrando un déficit de conocimientos tanto de los métodos diagnósticos como de los procedimientos básicos que se incluyen para la obtención de la muestra. Por otro lado, el hecho de que el 55\% de los estudiantes no haya participado en actividades de diagnóstico no permite conocer si estos tienen las competencias para dichas actividades.

Es necesario entonces buscar estrategias para mejorar las competencias cognitivas y técnicas relacionadas a la promoción y detección de la tuberculosis pediátrica, tanto en la formación del pregrado como en la realización del servicio social en los establecimientos de salud. A esto se debe agregar estrategias dirigidas al fortalecimiento de competencias para la realización de procedimientos básicos de enfermería que se incluyen en la obtención de muestras diagnósticas.

También es importante buscar mecanismos para lograr que el estudiante participe en las actividades de promoción y detección de la tuberculosis pediátrica, involucrando a las instituciones de servicio, para que las habilidades y destrezas del estudiante puedan perfeccionarse y que este pueda brindar atención de calidad al insertarse en el campo laboral como profesional de enfermería. 


\section{Referencias}

Hernández, R., Fernández, C., Baptista, M. (2014). Metodología de la investigación. McGraw-Hill/ Interamericana Editores, S.A. DE C.V.

Mellado, M., Santiago, B., Baquero, F., Moreno, D., Pineiro, R., Méndez, A., Noguera, A. (2017). Actualización del tratamiento de la tuberculosis en niños. Anales de pediatría, 88(1), 52. e152. e12. https://www.sciencedirect.com/science/article/pii/S1695403317302254

Ministerio de Salud. (2015). Plan estratégico nacional multisectorial para el control de la tuberculosis en El Salvador 2016-2020. MINSAL

Ministerio de Salud. (2017). Guía clínica para la atención pediátrica de la tuberculosis y la coinfección TB-VIH. http://asp.salud.gob.sv/regulacion/pdf/guia/guia_clinica_atencion_tb_v2.pdf

Ministerio de Salud (2019a). Descripción del programa de tuberculosis. https://www.salud.gob. sv/archivos/pdf/TUBERCULOSIS_DOC/descripcion_del_programa_TB102019.pdf

Ministerio de Salud. (2019b). Casos de VIH/SIDA hasta el 2018. Sala situacional de VIH/SIDA. https://datos.salud.gob.sv/vih/index.php/tabladinamica/casosnuevos

Ministerio de Salud. (2020a). Lineamientos técnicos para la prevención y control de la tuberculosis. http://asp.salud.gob.sv/regulacion/pdf/lineamientos/lineamientostecnicosparalaprevencionycontroldelatuberculosis-Acuerdo1513.pdf

Ministerio de Salud. (2020b). Norma técnica para la prevención y control de la tuberculosis. http://asp.salud.gob.sv/regulacion/pdf/norma/normatecnicaparalaprevencionycontroldelatuberculosis-Acuerdo1406.pdf

Ministerio de Salud. (2021). Guía clínica para la atención pediátrica de la tuberculosis y la coinfección TB-VIH. http://asp.salud.gob.sv/regulacion/pdf/guia/guia_pediatrica_tuberculosis_coinfeccion_tb_vih_v1.pdf

Organización Mundial de la Salud. (2016). Hoja de ruta para abordar la TB infantil: hacia cero muertes. http://apps.who.int/iris/bitstream/handle/10665/205064/9789243506135_spa.pdf;jsessionid=BE2F99CC0979A937F16DFF81F19472D8? sequence $=1$ 